\title{
Conditions de stabilité thermodynamique de la solution solide du système $\mathrm{CdTe}-\mathrm{HgTe}$
}

\author{
V.P. Vassiliev, I.V. Pentine et G.F. Voronine \\ Laboratoire de Thermodynamique Chimique, Faculté de Chimie, Université \\ Lomonossov, Moscou 119992, Russie
}

\begin{abstract}
The thermodynamic functions and the coordinates of the miscibility gap in the pseudo-binary system $\mathrm{Hg}_{1-\mathrm{x}} \mathrm{Cd}_{\mathrm{x}} \mathrm{Te}$ have been computed from published experimental data.
\end{abstract}

\section{INTRODUCTION}

Le cadmium, le mercure et le tellure forment sur la coupe quasibinaire $\mathrm{CdTe}-\mathrm{HgTe}$ une solution solide $\mathrm{Hg}_{(1-\mathrm{x})} \mathrm{Cd}_{\mathrm{x}} \mathrm{Te}(\mathrm{CMT})$ continue au-dessous du solidus pour $\mathrm{x}$ compris entre 0 et 1 .

La solution de composition $\mathrm{x}: 0.15 \leq x \leq 0.23$ est un matériau moderne utilisable pour la fabrication des détecteurs de rayonnement infrarouge à grandes longueurs d'onde [1,2]. La stabilité thermodynamique de la solution est une caractéristique importante puisqu'elle détermine les conditions de synthèse, d'exploitation et de durabilité du dispositif. Selon la thermodynamique, une solution solide dont la température baisse peut s'ordonner ou se décomposer en donnant les composants purs ou des composés définis.

Une démixtion de la solution CMT en deux solutions primaires a été mise en évidence expérimentalement pour la première fois par des mesures de potentiel chimique du cadmium dans des piles électrochimiques $[2,3]$ :

(-) Cd $\mid$ solution de glycérol $+\mathrm{CdCl}_{2} \mid \mathrm{Hg}_{(1-\mathrm{x})} \mathrm{Cd}_{\mathrm{x}} \mathrm{Te}+\mathrm{Te}(+)$ entre 340 et $440 \mathrm{~K}$

$(-) \mathrm{Cd} \mid$ fusion saline $+\mathrm{CdCl}_{2} \mid \mathrm{Hg}_{(1-\mathrm{x})} \mathrm{Cd}_{\mathrm{x}} \mathrm{Te}+\mathrm{Te}(+)$ entre 580 et $700 \mathrm{~K}$

Les points expérimentaux $\Delta \mu_{\mathrm{Cd}}(\mathrm{x}, \mathrm{T})$ sont présentés sur la Fig.1.

Le potentiel de la pile (I) pour les concentrations $x$ de 0.3 à 0.7 ne dépend pas pratiquement de la composition de la solution. Cela signifie que ces alliages appartiennent au domaine hétérogène. L'extrapolation des valeurs du potentiel de la pile (II) aux températures plus basses a permis d'évaluer les coordonnées du sommet de la lacune de démixtion avec $\mathrm{T}_{\mathrm{c}}=456.5 \mathrm{~K}$ et $x_{\mathrm{c}}=0.535$. Plus tard, ces résultats ont été confirmés par la calorimétrie différentielle à balayage (DSC) en mesurant les capacités thermiques des différents échantillons [4]. Leur chauffage depuis la température ambiante provoque une diminution irrégulière de la capacité thermique. Ce phénomène correspond au passage du système de l'état hétérogène à l'état homogène. Les paramètres du point critique $\left(T_{c}=455 \mathrm{~K}\right.$ et $\left.x_{c}=0.55\right)$ selon [4] ne se distinguent pas, pratiquement, de ceux de $[2,3]$.

Yu T.-C. et Brebrick R.F. [5, 6] proposent, à partir de mesures de pression partielle de vapeur de $\mathrm{Hg}, \mathrm{Cd}$ et $\mathrm{Te}_{2}$ par une méthode optique, $\mathrm{T}_{\mathrm{c}}=464$ et $454.7 \mathrm{~K}$, respectivement, $(x=0.5$ dans les deux cas). Des valeurs critiques $\left(\mathrm{T}_{\mathrm{c}}=494 \mathrm{~K}\right.$ et $\left.x_{\mathrm{c}}=0.6\right)$ ont été trouvées par la mesure des tensions de vapeur pour trois compositions $\mathrm{Hg}_{(1-\mathrm{x})} \mathrm{Cd}_{\mathrm{x}} \mathrm{Te}$ avec $\mathrm{x}=0.40,0.54$ et 0.70 [1]. Sidorko et Goncharuk [7] ont obtenu les valeurs du potentiel chimique de CdTe à la température $T=643 \mathrm{~K}$ pour 8 alliages ( $\mathrm{x}$ de 0.2 à 0.9 ) par la méthode FEM. Des mesures calorimétriques d'enthalpies de formation de trois alliages $\mathrm{Hg}_{(1-\mathrm{x})} \mathrm{Cd}_{\mathrm{x}} \mathrm{Te}$ pour $\mathrm{x}=0.6,0.705$ et 0.8 ont été faites par Rugg et al [8]. Malheureusement, les incertitudes de mesure sont comparables avec les valeurs enthalpiques elles-mêmes. Une des dernières optimisations des fonctions thermodynamiques de CMT a été présentée par Yang et al [9] avec des paramètres critiques pour la lacune de démixtion $T_{c}=600 \mathrm{~K}$ et 
$\mathrm{x}_{\mathrm{c}}=0.5$. L'analyse des propriétés thermodynamiques de la solution solide CMT telles qu'elles figurent dans la littérature démontre un manque de cohérence.

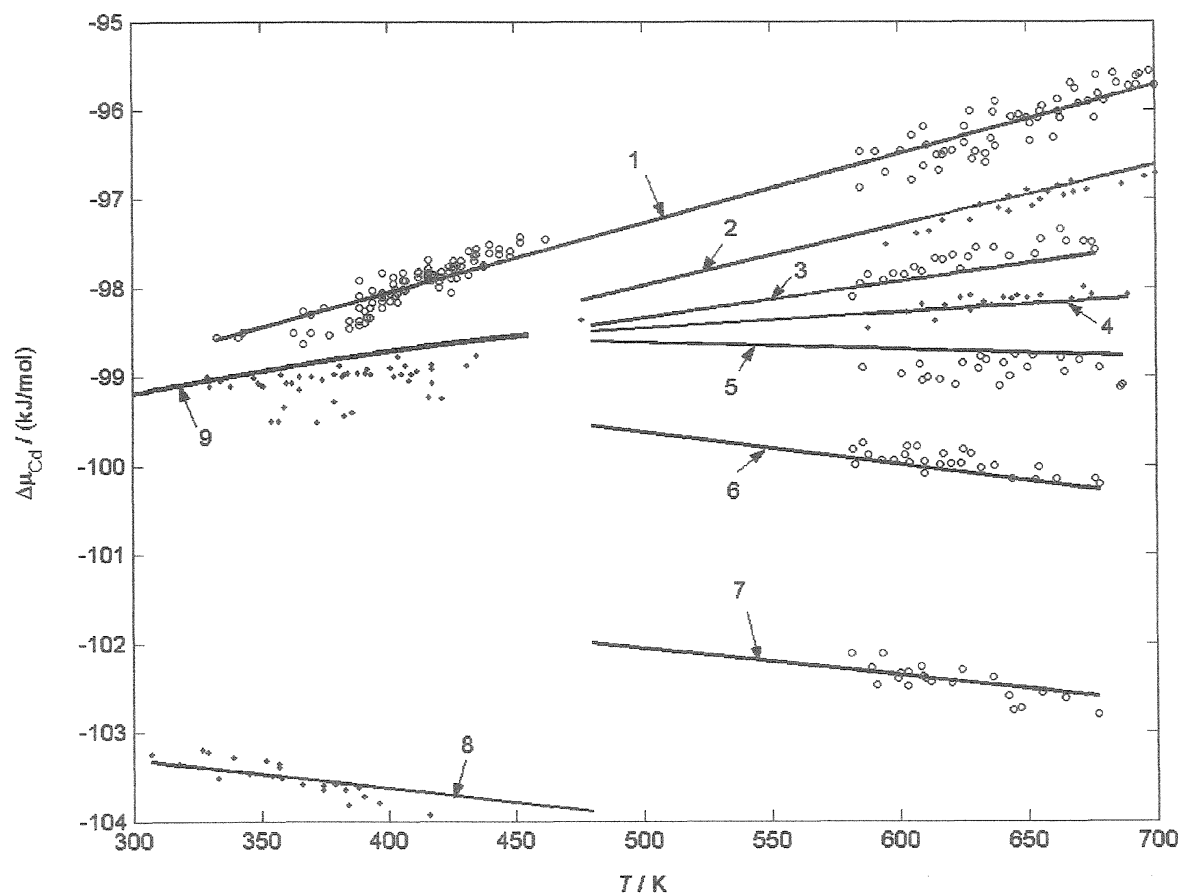

Figure 1 : Points expérimentaux [ce travail] et leur description à l'aide de l'équation (2). Les compositions des alliages $\mathrm{Hg}_{(1-\mathrm{x})} \mathrm{Cd}_{\mathrm{x}} \mathrm{Te}$ (séries 1 à 8 ) sont $x_{\mathrm{Cd}}=1,0.8,0.6,0.5,0.4,0.22,0.1$ et 0.06 , respectivement. La série 9 correspond au domaine hétérogène $\left(\mathrm{s}^{\prime}+\mathrm{s}^{\prime \prime}\right)$ pour les fractions molaires $0.3<x_{\mathrm{Cd}}<0.7$.

\subsection{Résultats}

On voit sur la Fig.2 (représentation $\mathrm{x}_{\mathrm{CdTe}}, \mathrm{T}, \Delta \mathrm{G}_{\mathrm{f}}$ ) un changement de concavité dans le domaine de la lacune. Dans ces conditions, l'extrapolation des fonctions thermodynamiques partielles vers le domaine de démixtion ne donne pas des résultats satisfaisants, bien que ces fonctions aient été mesurées avec une haute réversibilité (écart standard de $0.2-0.5 \%$ ). De plus, les propriétés thermodynamiques sont décrites séparément dans $[2,3,4]$ pour chaque composition de la solution. Un traitement sans précaution de toutes les données ne peut mener qu'à des incohérences. Le but de notre travail consiste à éliminer ces défauts.

Une bonne cohérence des résultats thermodynamiques est obtenue par la minimisation de la fonction:

$$
\sum \mathrm{w}_{\mathrm{i}}\left[\left(\mathrm{f}_{\text {calc }}-\mathrm{f}_{\text {mes } \mathrm{i}}\right) / \mathrm{f}_{\text {mes }}\right]^{2}=\min
$$

où $\mathrm{w}_{\mathrm{i}}$ sont les poids des ensembles de données. Ils sont choisis égaux à 1 pour les 8 ensembles de $[2,3]$ et à 0.1 pour [4]. Le symbole ' $f_{\text {mes }}$ ' représente les valeurs mesurées (la FEM des 8 ensembles $[2,3]$ et $\left.T_{\mathrm{c}}[4]\right), \mathrm{f}_{\text {cal }}$ les valeurs calculées.

L'optimisation donne une description de l'énergie libre de formation de la solution $\mathrm{Hg}_{(1-\mathrm{x})} \mathrm{Cd}_{\mathrm{x}} \mathrm{Te}$ : 


$$
\begin{aligned}
& \Delta \mathrm{G}_{\mathrm{f}}(\mathrm{T}, \mathrm{x})=\mathrm{x}(1-\mathrm{x})\left\{4617.31-2721.25(1-2 \mathrm{x})-6863.63(1-2 \mathrm{x})^{2}+[3.55132+4.62785\right. \\
& \left.\left.(1-2 \mathrm{x})+12.25412(1-2 \mathrm{x})^{2}\right] \mathrm{~T}\right\}+\mathrm{RT}[\mathrm{x} \ln (\mathrm{x})+(1-\mathrm{x}) \ln (1-\mathrm{x})](\mathrm{J} / \mathrm{mol})
\end{aligned}
$$

où $\mathrm{x}$ est la fraction molaire de CdTe. L' expression de l'énergie libre standard de la formation de CdTe est :

$$
\Delta \mathrm{G}_{\mathrm{f}}(\mathrm{T})=-101174.66+7.80732 \mathrm{~T}
$$

Les courbes binodale (4) et spinodale (5) calculées dans ce travail se décrivent au-dessus de $300 \mathrm{~K}$ par les équations suivantes :

$$
\begin{aligned}
& T(x) / \mathrm{K}( \pm 2.5 K)=T_{\mathrm{c}}\left(1-6.33867\left|x-x_{\mathrm{c}}\right|^{2.71702}\right) \\
& T_{s p}(x) / \mathrm{K}( \pm 2)=-46032.7 x^{4}+100289.4 x^{3}-83910.0 x^{2}+31819.3 x-4139.2
\end{aligned}
$$

Les paramètres du point critique sont : $T_{\mathrm{c}}=455.4 \mathrm{~K}, x_{\mathrm{c}}=0.53$

Les coordonnées des points spinodaux $T_{s p}(x)$ pour les températures données sont calculées à l'aide de l'équation $\left(\partial^{2} \Delta G_{f} / \partial x^{2}\right)=0$.

Chen K.-T., Wiedemeier [1], sur la base de leurs propres résultats, proposent une lacune de démixtion avec $T_{\mathrm{c}}=494 \mathrm{~K}$ et $x_{\mathrm{c}}=0.6$. Nous avons recalculé ces résultats et nous avons obtenu une lacune de démixtion plus large avec d'autres coordonnées du point critique. Dans ce cas, la limite de la zone de démixtion s'exprime par l'équation :

$$
T(x) / \mathrm{K}( \pm 3 K)=-5620.0 x^{4}+11899.7 x^{3}-9955.0 x^{2}+3929.3 x-137.7
$$

avec $\mathrm{T}_{\mathrm{c}}=478 \mathrm{~K}$ et $\mathrm{x}_{\mathrm{c}}=0.6$. Les courbes limites de démixtion sont présentées sur la Fig. 3 selon les différentes sources.

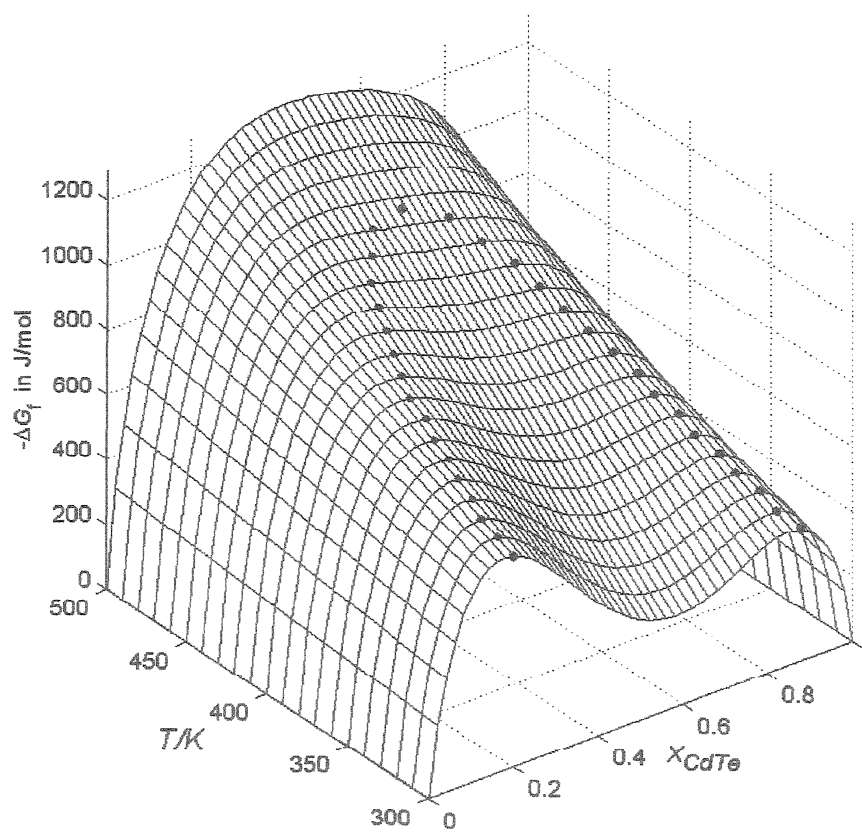

Figure 2 : Surface d'énergie libre de formation $\Delta G_{\mathrm{f}}(\mathrm{T}, x)$ de la solution solide $\mathrm{Hg}_{(1-\mathrm{x})} \mathrm{Cd}_{\mathrm{x}} \mathrm{Te}$. Les points sur la surface sont les coordonnées de la lacune. 


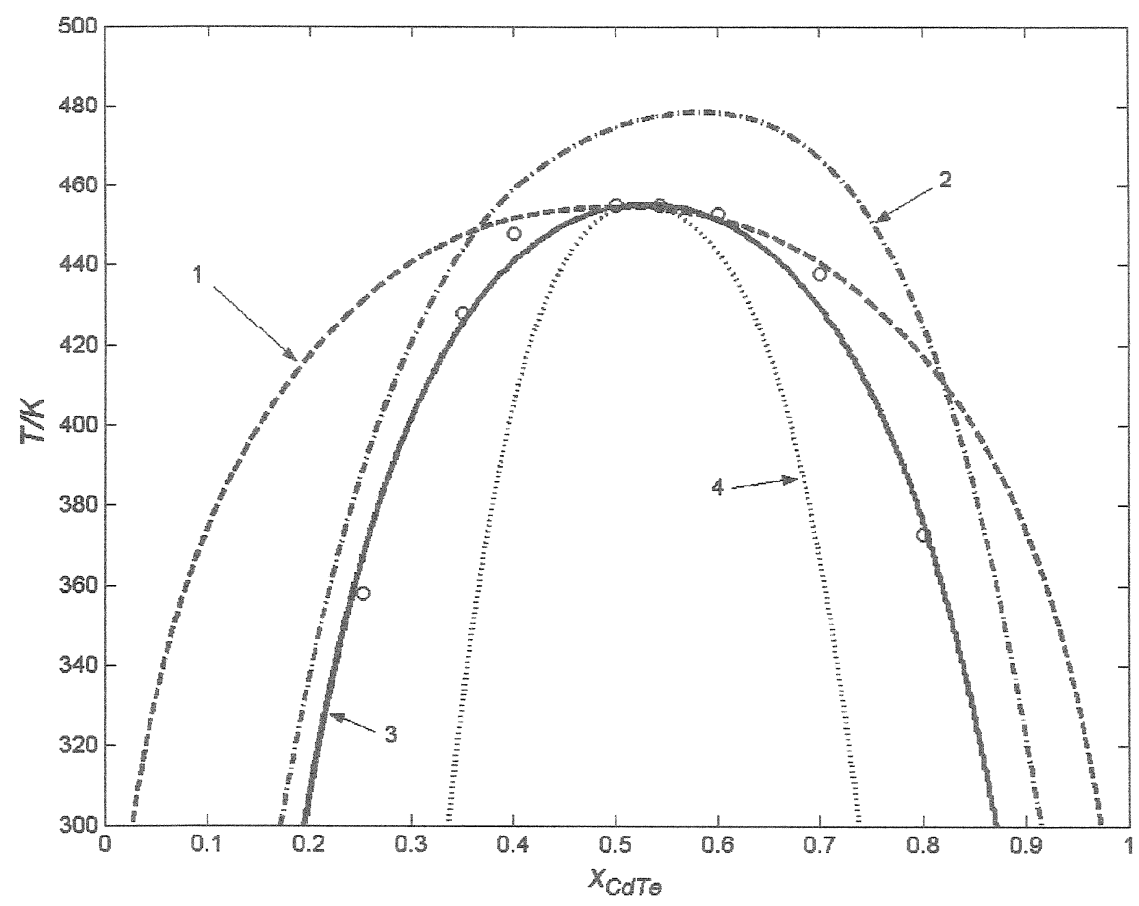

Figure 3 : Coordonnées de la lacune de démixtion $\mathrm{Hg}_{(1-\mathrm{x})} \mathrm{Cd}_{\mathrm{x}} \mathrm{Te}$.

Points expérimentaux [4], 1 : résultats [5], 2 : binodale recalculée selon les résultats [1], 3 et 4 : binodale et spinodale calculées (ce travail).

\section{CONCLUSION}

Une nouvelle description des fonctions thermodynamiques et un nouveau calcul de la lacune de démixtion de la solution solide $\mathrm{Hg}_{(1-\mathrm{x})} \mathrm{Cd}_{\mathrm{x}} \mathrm{Te}$ sont proposés. Les nouvelles coordonnées du point critique, calculées dans ce travail, ne se distinguent presque pas de [3, 4] puisque l'optimisation est basée sur ces résultats. Le même type de dépendance fonctionnelle des propriétés thermodynamiques de la solution solide est obtenue, mais avec d'autres paramètres numériques. Avec ces derniers, les erreurs de restitution des données initiales sont réduites d'un facteur 1.5-2.

\section{Références}

1. K.-T. Chen, H. J. Wiedemeier, Electron. Mater., 24, 405 (1995).

2. V.P. Vassiliev, M.N. Mamontov, V.V. Morozova, IV All-Union conference: Termodynamic and material research of semiconductors, part 1, Moscou,1989, p.97.

3. V.P. Vassiliev, M.N. Mamontov, M.A. Bykov Vestnik Moskovskogo Universiteta, serie2, Chimie, 31 (3) 211 (1990).

4. M. Gambino, V. Vassiliev, J.P. Bros, Alloys Comp. J., 176, 13 (1991).

5. T.-C. Yu, R.F. Brebrick, Phase Equilibria, 13 (5), 476 (1992).

6. T.-C. Yu, R.F. Brebrick, in Properties of narrow gap cadmium-based compounds. EMIS Data reviews Series №10, Ed. P.Capper, (INSPEC, IEE, 1994), p.55.

7. V.R. Sidorko, L.V. Goncharuk, Alloys Comp. J., 228, 13, (1995).

8. B.C. Rugg, N.S. Silk, A.W. Bryant, B.B. Argent, CALPHAD, 19 (3), 389, (1995). 